\title{
Naturalisierung der Freiheit aus Sicht der Verhaltensforschung ${ }^{1}$
}

\section{Naturgesetz und Zufall}

Im 19. Jahrhundert, als die Wirklichkeit noch als vollständig determiniert angesehen werden und man sich vorstellen konnte, dass es für alles lückenlose Ursachen gäbe, jede Veränderung also vom Beginn bis zum Ende des Universums im Prinzip zu jeder Zeit feststünde, war es naheliegend, ja vielleicht sogar denknotwendig, die Freiheit im Protest gegen diese Doktrin oder als „Alleinstellungsmerkmal“ des Menschen mit der Verletzung der Naturgesetze in Verbindung zu bringen. Da aber, wie uns die Physik des 20. Jahrhunderts lehrt, die Fäden von Ursachen und Wirkungen wegen der Quantenunbestimmtheit nicht von Anfang bis Ende durchgehen, sondern anfangen und abreißen, muss man heute die Verletzung der Physik als Bedingung für Freiheit nicht mehr fordern. Was immer wir unter Willensfreiheit verstehen, die Zukunft ist offen. Es kann objektiv einen Unterschied für den Lauf der Welt machen, ob wir uns Mühe geben oder nicht.

Mir ist bewusst, dass der Widerstand gegen die Ursachenlosigkeit unter den Philosophen immer noch groß ist. Aber die Annahme, man könne das Plancksche Wirkungsquantum unterlaufen, das heißt es gäbe unterhalb der Elementarteilchen noch eine weitere Ebene der Analyse, auf der man eines Tages zum Beispiel die konkrete hinreichende Ursache für das einzelne Ereignis im $\beta$-Zerfall werde finden können, ist in der Physik intensiv diskutiert worden. Man denke nur an Einsteins berühmtes „Gott würfelt nicht!“‘. Heute sind die meisten Physiker der Meinung, die Annahme einer solchen weiteren Ebene sei mit der Physik unvereinbar, und die Suche danach wurde eingestellt. Ich finde die Ursachenlosigkeit nicht so skandalös, wenn ich mir klar mache, dass die Physik eine Abstraktion ist, und mir die schier unendliche Kreativität dieses Daseins vergegenwärtige. Das verbissene Festhalten an der Vor-

Wiederabdruck des Beitrags von Martin Heisenberg in Naturgeschichte der Freiheit, hrsg. von Jan-Christoph Heilinger, Berlin, New York 2007, S. 43-57. 
stellung, nichts geschehe ohne hinreichende Gründe, ist das Relikt einer schon seit 100 Jahren überwundenen Fehlinterpretation der Naturwissenschaften. Ich werde jedenfalls die mögliche Ursachenlosigkeit von Ereignissen für meine weiteren Ausführungen voraussetzen. Freiheit macht nur unter dieser Prämisse Sinn.

\section{Zufall und Freiheit}

Erst wenn man den Determinismus ganz und gar abgeschüttelt hat, kann die Freiheit wieder selbstverständlich werden. Sie ist Teil unserer Wirklichkeit, eine Qualität des Lebens, ein Element unserer Existenz. Sie gibt es einfach, wie es Gedanken, die Temperatur oder das Licht gibt. So wie der Zufall an allen Vorgängen in der belebten und unbelebten Natur seinen Anteil hat, einmal mehr, einmal weniger, so haben die Lebewesen mehr oder weniger Anteil an der Freiheit. Wir schreiben Lebewesen Zustände zu, und zu den Eigenschaften solcher Zustände gehört die Freiheit. Man entlässt einen Vogel, der sich ins Haus verirrt hat, in die Freiheit. Man spürt die Freiheit nicht wie Kälte oder Schmerz, aber man reagiert auf den Mangel oder Überfluss dieser elementaren Bedingung unseres Daseins äußerst empfindlich. $\mathrm{Zu}$ viel und zu wenig davon können Gewalt, Angst oder Depression auslösen. Dem Gefühl der Freiheit lässt sich nicht immer trauen, aber eine Welt ohne Freiheit lässt sich nicht denken. Wäre denn zum Beispiel das Denken selbst ohne Freiheit möglich? Kann sich nicht jemand einfach Freiheit nehmen, außer man tötete ihn? Die Freiheit steht nicht zur Disposition, trotz der verbreiteten Zweifel unter Neurobiologen (vgl. Roth 2004; Singer 2006).

\section{Freiheit als Thema der Biologie}

Nicht erst die Soziologie, Pädagogik, Ökonomie oder Jurisprudenz, sondern auch die Humanbiologie würde ihrem Gegenstand nicht gerecht werden, wenn sie die Freiheit im menschlichen Verhalten auszuklammern oder zu leugnen versuchte. Schon in der Verhaltensbiologie der Tiere lässt sich die Freiheit nicht übersehen. Im Gegenteil: Aus diesem Thema kann man Gewinn für die biologische Forschung ziehen. Ich will im Folgenden Beispiele aus der Gehirn- und Verhaltensforschung schildern, die schon bei Tieren die Freiheit als wesentliches Element des Verhaltens erkennen lassen. Vielleicht können meine Ausführungen dazu beitragen, unter Biologen und Gehirnforschern die verbreiteten Missverständnisse über Freiheit zu beseitigen.

Wenn man den Determinismus nicht ganz abgeschüttelt hat, kann die Beschäftigung mit den Naturgesetzen leicht zu der Vorstellung führen, dass alles, was prinzipiell der naturwissenschaftlichen Betrachtung entgeht, nicht eigentlich Teil der Wirklichkeit sein könne. Aus dieser Einstellung resultiert m. E. oft die oben erwähnte Ablehnung der Freiheit unter Naturwissenschaftlern. 
Das Argument gegen die Freiheit scheint mir etwa so zu lauten: Alles Abwägen von Gründen geschieht im Gehirn. Dort gibt es Nervenzellen, Botenstoffe und Ionenströme. Was man mit diesem Inventar im Prinzip machen kann, wissen wir schon. Es kommen dabei nur Verursachungen vor, und sollte aufgrund der Quantenmechanik doch einmal etwas unverursacht geschehen, so wäre das der reine Zufall. Aber weder verursachte Handlungen noch solche, die durch einen Zufallsprozess im Gehirn ausgelöst wurden, bezeichnen wir als frei. Und etwas Drittes gibt es nicht. Zwischen Gesetzmäßigkeit und Zufall ist kein Platz für etwas Drittes. Wenn es nicht die Gründe sind, die einer der Handlungsalternativen den Zuschlag verschaffen, kann es nur der Zufall sein.

\section{Das Erlebnis der Freiheit ist keine Illusion}

Wer die Freiheit im Verhalten ablehnt, bezeichnet das Erlebnis der Freiheit in der Regel als Illusion (Wegner 2002). Illusionen kennen wir zum Beispiel aus Zaubervorstellungen oder aus der Sinnesphysiologie. Dort spielen sie eine wichtige Rolle. Wir bezeichnen die Deutungen von eigenen Erfahrungen als Illusionen, wenn sie im Widerspruch zu einem großen in sich konsistenten Verbund anderer Erfahrungsdeutungen stehen. So zum Beispiel die Halluzinationen, die sich mit unzähligen Erfahrungen anderer, wie auch mit anderen Erfahrungen der Patienten selbst, nicht in Einklang bringen lassen. So wird auch nicht das Erlebnis der Freiheit in Frage gestellt, sondern seine Deutung als wirkliche Freiheit in unseren Handlungen. Aber mit welchen anderen Erfahrungsdeutungen sollte die der relativen Handlungsfreiheit im Konflikt stehen? Die Erfahrung, dass nichts ohne Gründe geschehe, kann hier sicher nicht angeführt werden. Sie gibt es nicht. Das entsprechende Postulat ist ein theoretisches Konstrukt des 19. Jahrhunderts. ${ }^{2}$ Wir erleben oft Ereignisse ohne hinreichende Gründe und die Physik bescheinigt uns seit der Quantenmechanik, dass das keine Illusion sein muss. In Wirklichkeit ist die überwältigende Erfahrung, dass wir fast immer ohne hinreichende Gründe handeln. Unser Verhalten ist so „fehlerfreundlich“, dass selbst ein völlig unvorhersehbarer neuer Grund im letzten Moment noch berücksichtigt werden kann. So zufallsdurchtränkt wie die Ergebnisoffenheit ist auch schon das Abwägen von Gründen. Die Erfahrung von relativer Handlungsfreiheit kann also nicht als Illusion eingestuft werden, weil es keinen anderen Erfahrungsschatz gibt, zu dem sie in Widerspruch steht.

2 Ältere theologische Vorstellungen der Allmacht Gottes seien hier übergangen, so interessant sie in diesem Zusammenhang auch sind. 


\section{Naturgesetze und Einmaligkeit}

Die Naturgesetze sind nicht die Natur. Unser persönliches Dasein ist ein existenzielles Ereignis, ein Einzelfall. In den Umständen, in denen wir leben, sind wir mit der Bewältigung von lauter Einzelfällen beschäftigt. Unsere Wahrnehmungen und Gefühle, unsere Gedanken und Erinnerungen, unser Bewusstsein und unsere Freiheit sind zunächst solche singulären Lebensmomente. Sie gibt es einfach, wie es diesen Baum und jenen Bach, diese Wolke oder den Abendstern gibt. Das ist der metaphysische Hintergrund, vor dem sich das Dasein abspielt. Untrennbar davon, aber doch erst in zweiter Linie, können wir unser Dasein reflektieren, unsere Erfahrungen in Begriffe fassen, darüber nachdenken, miteinander reden und schließlich auch Wissenschaft betreiben.

Selbst wenn in der naturwissenschaftlichen Mikroanalyse der Handlungsfreiheit nur Verursachungen und Zufälle auftreten, ist die Handlungsfreiheit deswegen nicht weniger wirklich. Auch bei der Mikroanalyse der Temperatur finden wir nur die Bewegung von Atomen und Molekülen. Wer würde behaupten, dass es die Temperatur deswegen in Wirklichkeit nicht gäbe. Dann könnte man uns ja auch den Schmerz ausreden, der ja ,in Wirklichkeit“ nur eine Kette physiologischer Vorgänge im Gehirn sei. Handlungen können in Wirklichkeit unfreie und freie Handlungen sein.

An dieser Stelle sei erwähnt, dass viele Menschen immer noch eine Verletzung der Physik als notwendige Bedingung von Handlungsfreiheit ansehen. Es macht m. E. keinen Sinn anzunehmen, wir könnten mit jedem Willensakt die Physik aus den Angeln heben. Mir ist noch kein seriöser Gehirnforscher begegnet, der zur Begründung der Handlungsfreiheit im Gehirn nach Verletzungen der Physik sucht. Wir nehmen immer nur Verursachungen und Zufälle an. Eine Verletzung der Physik ist, wie gesagt, seit der Quantenmechanik nicht mehr nötig.

Um diese kurze Positionierung zusammenzufassen: Die Willensfreiheit lässt sich nicht durch naturwissenschaftliche Befunde im Gehirn widerlegen. Freiheit hat mit Risiko und Möglichkeit zu tun. Es geht darum, dass wir, die Akteure, existenzielle Subjekte sind. „Bin so frei!“ sagt der Wiener.

Was ist diese Qualität menschlichen Verhaltens, die wir Freiheit nennen? Ich will vier Aspekte nennen: Die Urheberschaft, das Wollen, die Entscheidung und die Gedankenfreiheit. In der Verhaltensbiologie oder Bio-Psychologie können alle diese Aspekte untersucht werden. Es versteht sich, dass wir im Gehirn immer nur Verursachungen und Zufälle am Werk finden werden, vermutlich in faszinierendem Zusammenspiel. Dieses Zusammenspiel könnte sich bei der ergebnisoffenen Abwägung von Gründen in menschlichen Angelegenheiten als unentwirrbar erweisen. Ich will aber auch nicht bei den Psychologen ,wildern“, sondern lieber Beispiele aus meinem Erfahrungsbereich bringen - der Gehirnforschung an der Fliege Drosophila. 
Zwei so wichtige Elemente unserer Freiheit wie die Urheberschaft und die Entscheidung findet man schon bei den niederen Tieren.

\section{Urheberschaft}

Würde nichts ohne hinreichende Ursachen geschehen, wäre es unsinnig, jemandem Urheberschaft zuzuschreiben. Wo sollte man die unendliche Kette der Verursachungen durchtrennen? Ist jedoch die Ursachenlosigkeit erst einmal zugestanden, können die Gründe vollständig im jeweiligen Individuum liegen. Mit dieser theoretischen Klarstellung wollen wir uns dem raffinierten Gemisch von Gründen und Zufällen zuwenden, das wir Urheberschaft nennen. Dazu muss ich weit ausholen.

Die Gene sichern den Entwicklungsweg vom befruchteten Ei bis zum ausgereiften Gehirn. Viele Gene sind dabei unentbehrlich, ohne sie bricht die Entwicklung zusammen. Man kann vom genetischen Entwicklungsprogramm sprechen, wenn man dabei zum Beispiel an das Programm eines Kindergeburtstags denkt. Der Ablauf muss immer wieder kontrolliert, gebündelt, stabilisiert und mit Information versorgt werden. Im genetischen Programm der Gehirnentwicklung gibt es aber auch Flexibilität und Freiräume. Eine durch einen Unfall abgestorbene Nervenzelle kann unter Umständen durch eine andere ersetzt werden. Der Embryo passt sich frühzeitig an die jeweiligen Lebensumstände an und in jeder Entwicklung sorgen kleine und größere Zufälle für die Einmaligkeit des jeweiligen Lebewesens, selbst bei eineiigen Zwillingen mit gleichem Erbgut. Die Gene sind weitgehend dafür zuständig, dass aus dem Ei einer Meise wieder eine Meise entsteht, aus dem eines Finken ein Fink. Im Zeitalter der Genomik haben die Gene viel von ihrem mystischen Flair verloren. Im Prinzip glauben wir zu verstehen, was die Gene für das Gehirn und das Verhalten leisten. Jedes Gen ist die Bauvorschrift für ein oder mehrere Proteine. Diese bestimmen direkt oder im Verbund mit dem Stoffwechsel die Eigenschaften der Zellen. Mit der Entstehung höherer Lebewesen haben die Zellen vielfältige Formen der Kommunikation untereinander entwickelt. Viele Nervenzellen sind zusätzlich auf besonders rasche Signalübertragung im Bereich von Millisekunden spezialisiert.

Eine der Errungenschaften dieser Kommunikation sind Nervennetze aus einigen Dutzend oder einigen tausend Zellen, die Sequenzen von Muskelaktivierungen programmieren können, die der Verhaltensforscher als Verhaltensmodule kennt, wie das Greifen des Säuglings, die Silben des Vogelgesangs oder die rhythmische Bewegung der Beine beim Laufen. Manche Module, wie der Herzschlag, dauern von der Embryonalzeit bis zum Tod. Andere, wie das Zuschlagen der Kiefer des Krokodils, dauern nur Bruchteile von Sekunden. Einige können parallel zueinander ablaufen, wie Gehen und Singen, einige verhindern sich gegenseitig, wie Schlafen und Klavier spielen, wieder einige schließen notwendig aneinander an, wie das Landemanöver 
des Vogels, der nur so das Fliegen beenden kann, usw. Das Leben von Tieren und Menschen ist von Anfang bis Ende ein fortlaufendes Geflecht solcher Verhaltensmodule. Viele dieser Verhaltensakte sind stereotyp, wie die Lautmuster der meisten Insekten und vieler Vögel. Andererseits können auch Tiere schon lernen, Verhaltensmodule zu Sequenzen zusammenzusetzen und ihren Ablauf zu perfektionieren.

Andere Nervennetze kontrollieren das Auftreten und die Feinstruktur dieser Bausteine, indem sie zum Beispiel Hormone in die Körperflüssigkeiten oder Modulatoren direkt in das Nervensystem abgeben. Wieder andere bereiten die Kontrollnetzwerke mit vorverarbeiteten Daten auf ihre Kontrollfunktionen vor und passen das ganze System mittel- und langfristig den Umständen und den damit verbundenen wechselnden Erwartungen an die Zukunft an. Diese Kontrolle ist weich, das heißt sie verändert die Wahrscheinlichkeiten für das Auftreten der Verhaltensmodule.

Eine der ursprünglichsten Wurzeln der Verhaltensfreiheit ist der hohe Grad der Autonomie von Lebewesen. Fast alle Prozesse, die in einem Organismus ablaufen, beziehen sich auf den Organismus selbst. Der Austausch mit der Umgebung erscheint im Vergleich dazu zweitrangig. Eine kleine Veränderung der Temperatur führt zu einer Fülle von Vorgängen im Organismus, die dafür sorgen, dass ihm daraus kein Nachteil erwächst. Organismen unterhalten Heerscharen von Schutzmaßnahmen gegen Fremdeinwirkung, nicht nur als Reaktionen, sondern auch als Vorsorge. Ein Beispiel für diese Autonomie ist die Fähigkeit, zwischen selbst- und fremdverursachten Sinnesreizen zu unterscheiden. Die Autonomie ist der entscheidende Grund, warum Organismen Urheber sein können. Sie können von sich aus Verhaltensmodule aktivieren, ohne Anstoß von außen. Für das Gehirn gilt in ganz besonderem Maße, dass es vorwiegend mit sich selbst beschäftigt ist. Diese initiale Aktivität werden wir gleich noch ausführlicher betrachten.

Hinsichtlich ihrer Verhaltensfreiheiten stellen autonome Wesen höchst spezifische Ansprüche an ihr Erbgut. Genetische Fehler können die Autonomie einschränken, die Menge der Verhaltensoptionen verkleinern, aber auch das Verhalten erratischer machen und damit die Qualität des Entscheidungsprozesses beeinträchtigen. Mit anderen Worten, unsere Gene ermöglichen einerseits überhaupt erst unsere Freiheit, andererseits begrenzen sie sie und können sie in krassen Einzelfällen auch weitgehend verhindern.

\section{Über die Freiheit des Ortes}

Kaum etwas hat das Verhalten so sehr geformt wie die Fortbewegung im Raum. Als Hinweis auf die Verhaltensfreiräume, die sich durch die Fortbewegung auftun, wird Niels Bohr sinngemäß mit dem Ausspruch zitiert, man könne kein Auto bauen, das nur von Hamburg nach Bremen zu fahren in der Lage wäre. Die Freiheit des 
Ortes geht vermutlich bis auf die Frühphase der Evolution zurück, als der Wettbewerb zwischen chemischen Verbindungen durch den Wettbewerb zwischen Membranbläschen abgelöst wurde, die frei im Wasser der Urmeere suspendiert waren. Die Tiere haben dieses Prinzip der örtlichen Ungebundenheit nicht aufgegeben, im Gegensatz zu den ortsfesten Pflanzen. Dadurch kann sich für die Tiere einerseits die Umgebung viel schneller und radikaler ändern als für Pflanzen, andererseits besitzen sie ein einzigartiges Mittel dieser Herausforderung zu begegnen: die aktive Fortbewegung. An die Freiheit des Ortes lassen sich die beiden Fragen „Frei wovon“ und „Frei wozu“ sinnvoll stellen. Tiere sind frei von einem bestimmten Ort, und sie sind in der Lage, Orte aufzusuchen. Die Struktur des Raumes bedingt, dass kleinste Unterschiede in der Richtung nach einer gewissen Zeit der Vorwärtsbewegung zu weit auseinander liegenden Orten führen.

Tiere sind im Prinzip aktiv, und nur deswegen können sie auch passiv sein. Unsere Sprache verfügt über eine aktive Form des Verbs, die in der Regel angibt, dass ein Wesen aus seiner Autonomie heraus und von sich aus initial etwas tut (Heisenberg 1983). Die Amsel pickt an einem Wurm, die Schildkröte springt ins Wasser. Unter dem Einfluss des Determinismus war aus der initialen Aktivität heimlich eine Reaktivität gemacht worden. Nach dem Motto: „Von nichts kommt nichts!“ hatte die Verhaltensforschung versucht, die initiale Aktivität als mangelndes Wissen des Beobachters wegzuerklären. Aber so wie heute noch die Motorik ihre Unabhängigkeit von der Sensorik dadurch dokumentiert, dass sie jener in der Entwicklung ein wenig vorausgeht, so war vermutlich auch in der Naturgeschichte die Entwicklung der Motorik von der Sensorik zunächst unabhängig, weil schon allein die Verbreitung im Raum aktive Mobilität evolutionär begünstigte. Wie dem auch immer gewesen sein mag, die initiale Aktivität ist ein Grundelement der Freiheit des Ortes und überhaupt der Verhaltensfreiheit.

Verhaltensmodule können, wie gesagt, ohne Anstoß von außen aktiviert werden (Heisenberg \& Wolf 1979), was nicht heißt, dass diese Aktivierung keine physiologischen Ursachen hätte. Nur muss sie auch ein Element des Zufalls enthalten. Die Aktivierung antwortet nicht auf einen Reiz oder ein anderes Verhalten, sondern sucht nach einer Antwort, nach noch unbekannten Wirkungen, die neue Möglichkeiten eröffnen. Verhalten $u m z u \ldots$, das nennt man im Fachjargon operant. Darunter fallen die verschiedensten Formen des Suchens und Ausprobierens. Operantes Verhalten gehört zu den elementarsten Grundlagen des Verhaltens überhaupt (Wolf \& Heisenberg 1991).

Im Ausprobieren erklärt sich das Verhalten nicht aus seinen Ursachen, sondern aus seinen Konsequenzen. Das Tier muss seinen Zustand vor und nach dem Verhaltensakt mit einem erstrebten Zustand vergleichen. Verringert sich der Abstand zwischen dem aktuellen Zustand und dem erstrebten, setzt das Tier das Verhalten fort, ver- 
größert er sich, bricht es das Verhalten ab und probiert etwas anderes. Beim Ausprobieren kann das Tier etwas über die Folgen seines Verhaltens lernen und damit unter Umständen später unangenehmen Situationen zuvorkommen. Diese Leistung wird operante Konditionierung genannt und kann bei Drosophila in den verschiedensten Versuchsbedingungen studiert werden.

\section{Entscheidung}

Man kann der Meinung sein, schwierige moralische Entscheidungen treffen zu können sei eine rein menschliche, soziokulturelle Leistung. Wenn man hier die Betonung auf die Moral legt, mag das so zutreffen. Trotzdem muss gelten, dass unser Gehirn das Abwägen von Gründen ermöglicht. Wir können also - mit Blick auf eine mögliche Naturgeschichte der Freiheit - die Verhaltensbiologie fragen, was sie über die Entscheidung weiß. Die Antwort ist überraschend: Die Organisation der Entscheidung ist stammesgeschichtlich vermutlich so alt, dass wir sie fast überall im Tierreich vorfinden. Tiere können nicht nur Verhaltensmodule initial aktivieren, sondern auch Vorgänge im Gehirn, so zum Beispiel Repräsentationen der möglichen Folgen von Verhaltensoptionen, um sie mit möglichen Folgen anderer Verhaltensoptionen zu vergleichen. Ein Beispiel, aus dem hervorgeht, dass schon im Drosophila-Gehirn Verhaltensmodule angeborenermaßen mit ihren möglichen Konsequenzen repräsentiert sind, ist die sogenannte schnelle Phototaxis, die Flucht zum Licht.

Wenn nichts Dringendes ansteht, putzen Fliegen sich. Dabei spielen die Flügel eine wichtige Rolle; stäubt man sie ein, verlängert sich das Flügelputzen - Flugbereitschaft ist offenbar sehr wichtig. Warum rennen die Fliegen in engen, dunklen Röhren zum Licht, wenn man sie erschreckt? Vermutlich, weil sie dort wegfliegen können. Auf dem Boden, im Dunkeln lauert Gefahr. Sie verraten uns mit ihrer Flucht zum Licht, dass ihnen die Konsequenzen des eigenen Verhaltens ,einprogrammiert“ sind. Diese Schlussfolgerung wird erst so richtig deutlich, wenn man die Flügel mit einem winzigen Tropfen Zuckerwasser oder Klebstoff zusammenklebt. Das Laufen wird dadurch nicht beeinträchtigt. Aber die Flucht zum Licht findet nicht statt. Egal wie man den Gebrauch der Flügel verhindert, durch Amputation, Mutationen in den Flugmuskel-Proteinen oder Blockade des Flugkontrollnetzwerks, wenn das Fliegen nicht funktioniert, flüchtet die Fliege nicht zum Licht, und sie hat auch keinen Anlass das zu tun (erwähnt in Heisenberg \& Wolf 1984).

Entscheidungen setzen also offenbar initiale Gehirnaktivität voraus. Diese kann man bei Drosophila direkt sichtbar machen: Die Fliege kann zum Beispiel ihre Aufmerksamkeit selektiv auf bestimmte Stellen in ihrem Sehfeld lenken. Meist folgt sie mit ihrer Orientierung rasch nach. Aber das können wir experimentell verhindern. Im folgenden Versuch hängt sie starr an einem Messgerät, das die Drehmomente 
aufzeichnet, mit denen sie im freien Flug ihre Rechts- und Linkskurven ausführen würde. Wenn man in dieser Situation links von der Fliege einen schwarzen Balken hin und her bewegt, versucht die Fliege dieser Bewegung mit charakteristischen Manövern zu folgen, die sich in den beiden Phasen, in denen der Balken sich mit der Flugrichtung der Fliege beziehungsweise gegen sie bewegt, unterscheiden. Wiederholt man das Experiment auf der anderen Seite, beobachtet man das entsprechende Verhalten, welches sich im Drehmoment natürlich spiegelsymmetrisch zeigt. Nun kommt der entscheidende Versuch: Wir präsentieren der Fliege jetzt zwei Balken, einen links, einen rechts, symmetrisch zur Mittelachse, und bewegen diese Balken im Gleichtakt hin und her, immer zusammen nach vorn und nach hinten. Was macht die Fliege? Sie bezieht sich mit ihren Flugmanövern eine Weile lang auf den einen Balken, dann auf den anderen. Sie blendet wechselnde Teile des Sehfeldes aus (Wolf \& Heisenberg 1980). Das Fliegengehirn generiert also von selbst, das heißt unabhängig vom Verhalten und von spezifischen Sinnesreizen, hoch geordnete, verhaltensrelevante Aktivität.

\section{Ziele}

Wenn Tiere ausprobieren, haben sie auch Ziele. Damit können Tiere, wie wir, Absichten und Wünsche, vielleicht auch in bescheidenem Maße Hoffnungen haben. Der Regenwurm probiert aus, wo er am besten durch das Erdreich dringt, der Vogel, ob ein neuartiges Material sich für sein Nest eignet. Dabei muss dem Vogel ein Ziel vorschweben, so primitiv diese „Vorstellung“ sein mag. Auch die Fliege Drosophila probiert aus, wie sie zum Beispiel eine bekömmliche Umgebungstemperatur finden oder ihren Flug stabilisieren kann. Das wird in einem Versuch besonders deutlich, der ursprünglich in der Humanpsychologie entwickelt worden ist und dort Berühmtheit erlangt hat.

Es ist das sogenannte Umkehrbrillen-Experiment. Die Probanden müssen eine Brille tragen, die zum Beispiel rechts und links vertauscht, und müssen damit zu leben lernen. Am Anfang des Experiments werden sie von Helfern geführt, bis sie in der Lage sind, die krassesten Fehler in ihrer visuomotorischen Koordination zu vermeiden. Stellen Sie sich vor, Sie trügen eine solche Brille. Sie stünden hier und wollten sich einer freundlichen Dame links von Ihnen zuwenden. Die sähen Sie jedoch mit der Umkehrbrille auf der rechten Seite und während Sie sich dorthin wendeten, verschwände sie rechts aus Ihrem Blickfeld. Das Experiment ist so berühmt, weil man sich an die Brille gewöhnt. Nach ein paar Tagen mit sehr unangenehmen Erlebnissen hört man auf, sich daran zu stören, dann merkt man nicht mehr, dass rechts und links vertauscht sind, außer wenn man danach gefragt wird, und schließlich, nach einer Woche, ist links wieder links und rechts rechts, selbst wenn 
man auf die Brille aufmerksam gemacht wird. Was lehrt uns dieses Experiment? Zum Beispiel, dass die Wahrnehmung ein ganzheitlicher Vorgang ist. Der Sehsinn ist im Streben nach erfolgreicher Orientierung im Raum und nach Konsistenz der Summe der anderen Erfahrungen untergeordnet.

Mit den Fliegen kann man ein ganz ähnliches Experiment durchführen. Wir hängen sie wieder an besagtes Messgerät, wo sie sich nicht drehen können, ihre Drehversuche aber aufgezeichnet werden. Aber jetzt bauen wir das Messgerät zu einem Flugsimulator aus. Ein Computer errechnet aus den Messwerten, wie schnell sich die Fliege drehen würde, wenn sie frei wäre und bewegt das Panorama, in dem die Fliege hängt, entsprechend in die andere Richtung. Im Flugsimulator führt also ein Drehversuch nach rechts zu einer Drehung des Panoramas nach links und umgekehrt. Dann setzen wir der Fliege im Flugsimulator sozusagen die Umkehrbrille auf. Das geht mit einem einfachen Schalter in der Steuerungselektronik. Jetzt führt plötzlich ein Drehversuch der Fliege nach rechts zu einer Drehung des Panoramas ebenfalls nach rechts und damit passiert der Fliege das, was vorhin dem Herrn auf dem Podium mit der Dame im Zuschauerraum passiert ist. Die Fliege versucht, sich auf eine Landmarke zuzudrehen, und diese verschwindet auf der gleichen Seite nach hinten aus dem Blickfeld.

Auch die Fliege lernt, sich mit ihrer neuen Situation zu arrangieren und nach ca. 40 Minuten ist sie wieder „Herrin der Lage“ (Heisenberg \& Wolf 1984). Sie hat gelernt, dass sie sich der Landmarke zuwenden kann, wenn sie das entgegengesetzte Flugmanöver macht, das sie normalerweise durchführen würde. Das konnte sie nur durch Ausprobieren herausfinden. Setzt man ihr die Umkehrbrille wieder $a b$, ist sie für einen kurzen Moment verwirrt, kehrt dann aber rasch in ihre normale Verhaltensweise zurück. Auf die Frage, wo im Gehirn die initiale Aktivität entsteht, die dem Tier das Ausprobieren ermöglicht, sind wir noch auf Spekulation angewiesen. Im Verdacht steht jedoch eine Region in der Mitte des Gehirns, der sogenannte Zentralkomplex.

Wie reagiert eine Fliege, der man die Entscheidung schwer macht? Fliegen im Flugsimulator wurden mit Bestrafungen konditioniert, bestimmte Flugrichtungen relativ zu Landmarken zu vermeiden. Die Landmarken unterschieden sich durch ihre Form und ihre Farbe. Ein blaues „T“ etwa war „gefährlich“, ein umgedrehtes grünes „T“ „sicher“. Das konnten die Fliegen rasch lernen, und schon eines der beiden Parameter, Form oder Farbe, reichten aus, der Fliege die sichere Flugrichtung zu weisen. Nun wurden aber für den Test die Formen und Farben umgekehrt kombiniert (Tang $\&$ Guo 2001). Auf einmal waren die aufrechten Ts grün und die umgedrehten blau. Was tun? Die Fliegen entschieden sich einmal so, das nächste Mal anders. Das Experiment war so eingerichtet, dass im Mittel alle Fliegen zusammen in ihrem Verhalten die erwartete Pattsituation widerspiegelten. Das eigentlich Interessante an diesem 
Experiment zeigte sich, als die Wissenschaftler die Reizstärke der Formen oder Farben im Test variierten. Sobald die Farben auch nur eine Nuance weniger rein waren, richteten sich die Fliegen nach den Formen, wenn dagegen die Formen auch nur eine Spur weniger unterschiedlich waren, richteten sich die Fliegen nach den Farben. Entgegen aller Erfahrungen aus früheren Versuchen zogen die Fliegen in diesem Konfliktfall auf einmal die Reizstärken der Testmuster für ihre Entscheidung mit heran. Die Zuverlässigkeit der Reize, die ohne die Pattsituation fast belanglos gewesen wäre, bekam eine entscheidende Bedeutung. Dieses Beispiel beleuchtet ein Funktionselement der Entscheidung, das sich offenbar bei Fliegen wie Menschen findet: In schwierigen Entscheidungssituationen werden weitere Kriterien in die Abwägung einbezogen. Man kann ahnen, welche interessanten Zusammenhänge uns erwarten, wenn wir eines Tages in die Verhaltensbiologie der Entscheidung tiefer eindringen können. Man wird die wichtigsten Kriterien finden, die die Philosophen an eine freie Entscheidung stellen: Keine Fremdbestimmung und keine unmittelbaren Sachzwänge, das heißt es muss Verhaltensoptionen mit hinreichend günstiger Prognose geben. Zu einer freien Entscheidung gehört auch, dass sie ohne zu große Fehler und Nachlässigkeiten in den Abwägungsvorgängen zustande kommt.

\section{Andere Verhaltensfreiheiten des Menschen}

Andere Verhaltensfreiheiten - wie das Wollen und die Gedankenfreiheit - habe ich hier ausgeklammert. Beide fallen nicht in meine Fachkompetenz. In beiden wird ein anderes Verhaltenselement zentral, der soziale Bezug. Wenn wir nach dem „Frei wovon“ und „Frei wozu“ fragen würden, müssten wir über die Beziehungen in der Gruppe und in größeren Sozietäten reden. Aber beide Themen zeigen die Wucht, die von dem in die Welt gesetzten Verhaltensakt ausgehen kann. Des Menschen Wille ist sein Himmelreich. Er versetzt Berge. Die Trennung von Wollen und Tun wird schon bei unseren Haustieren deutlich, beispielsweise wenn eine Katze sich überlegt, ob sie auf eine Mauer springen soll. In der Sozietät ist der Wille des Einzelnen zunächst nur eine Stimme in einem Konzert von Willensäußerungen. Um seinen Willen durchzusetzen, muss man sich in dieses Kräftespiel einfügen. Wer seinen Willen in der Gruppe durchgesetzt hat, trägt eine erhöhte Verantwortung für das Gelingen der gemeinsamen Aktion. Das Wollen ermöglicht einem die nachträgliche (wenn auch nicht reale, so doch mentale und soziale) Revision der Entscheidung. Man kann unterscheiden, was man tun wollte und was man getan hat. Genauer: In der Rekapitulation der Handlung verschafft einem die Trennung von Wollen und Tun die Möglichkeit, die Entscheidung im Licht ihrer Konsequenzen noch einmal zu vollziehen. 
Wieder ein ganz neues Kapitel ist die Gedankenfreiheit. Philipp II. meinte zu wissen, warum er in seinem Reich keine Gedankenfreiheit zulassen konnte. Einmal in die Welt gesetzt, lassen sich Gedanken unter Umständen nicht mehr unterdrücken. Aber die meisten hinterlassen kaum eine Spur, sind verflogen, noch ehe sie zur Rede gerinnen konnten. Wie frei sind Gedanken? Woher kommen sie? Woher kommt der Einfall, von dem der, der ihn hat, selbst überrascht wird? Gibt es Gesellschaften, in denen zwar Meinungsfreiheit, aber keine Gedankenfreiheit herrscht? Beide Themen, die Willens- und die Gedankenfreiheit, könnten wie die Entscheidungsfreiheit zentrale Themen der Gehirn-, Verhaltens- und Psychobiologie sein.

\section{Zusammenfassung}

In der Freiheit finden wir uns vor. Wir können versuchen, die biologischen Voraussetzungen dieses Befundes zu verstehen. Die Diskussion der letzten Jahre über Handlungs- und Willensfreiheit ist von den Nachwirkungen des Determinismus geprägt. Meine Pointe ist, dass wir mit der Überwindung des Determinismus nicht mehr um die Ursachenlosigkeit im Verhalten kämpfen müssen, sondern gerade das äußerst subtile Gefüge von Ursachen und Zufällen zum Thema machen können. Freiheit ist möglich, weil sie der Physik und Chemie des Gehirns nicht widerspricht. Als Beispiele für Verhaltensfreiheiten habe ich Urheberschaft, Wollen, Entscheidung und Gedankenfreiheit erwähnt, mich dann aber nur mit der Urheberschaft und der Entscheidung beschäftigt. Die Urheberschaft verdankt sich dem hohen Autonomiegrad tierischer Organismen. Die Entscheidung ist dadurch gekennzeichnet, dass dem Individuum Verhaltensoptionen offen stehen, zwischen denen es selbst abwägen muss. Abwägen zwischen Verhaltensoptionen bedeutet, dass die möglichen Folgen der zur Verfügung stehenden Verhaltensweisen repräsentiert, aktualisiert, verglichen und bewertet werden und dass die initiale Aktivierung des Verhaltens von dieser Abwägung abhängig gemacht wird. Die Entscheidungsfreiheit ist eine Freiheit von Fremdeinflüssen, die für eine verantwortliche Güterabwägung genutzt wird. Alle diese Eigenschaften kann man im biologischen Zusammenhang diskutieren und an Tieren beobachten. In der allgemeinen Organisation tierischen Verhaltens zeigen sich die Urheberschaft und die Grundelemente der Entscheidung. Schon niedere Tiere sind initial aktiv und können ausprobieren. Sie haben Ziele und Verhaltensoptionen, die in ihrem Gehirn durch ihre möglichen Folgen repräsentiert sind.

Freiheit ist überall im Verhalten von Tieren und Menschen zu entdecken. Sie ist für die Organisation des Verhaltens so wichtig, dass die Verhaltensforschung nicht umhin kann, sie zu thematisieren, auch wenn die Freiheit uns dabei auf der Mikroebene nur in unpersönlicher Verkleidung als Zufall entgegentritt. Die naturwissenschaftliche Erforschung der (naturalisierten) Freiheit wird unser Erleben der Freiheit beeinflussen. Wir werden klarer sehen, was Handeln frei macht. 


\section{Bibliographie}

Heisenberg, Martin (1983): Initiale Aktivität und Willkürverhalten bei Tieren. In: Naturwissenschaften 70, S. 70-78.

Heisenberg, Martin \& Reinhard Wolf (1979): On the Fine Structure of Yaw Torque in Visual Flight Orientation of Drosophila melanogaster. In: Journal of Comparative Physiology A (130), S. 113-130.

Heisenberg, Martin \& Reinhard Wolf (1984): Vision in Drosophila. Genetics in Microbehavior, Berlin, Heidelberg, New York: Springer.

Roth, Gerhard (2004): Das Problem der Willensfreiheit aus Sicht der Hirnforschung. In: Debatte 1, Zur Freiheit des Willens, S. 83-92.

Singer, Wolf (2006): Neurobiologische Anmerkungen zum Freiheitsdiskurs. In: Debatte 3, Zur Freiheit des Willens II, S. 17-26.

Tang, Shiming \& Aike Guo (2001): Choice Behavior of Drosophila Facing Contradictory Visual Cues. In: Science 294, S. 1543-1547.

Wegner, Daniel M. (2002): The Illusion of Conscious Will, Cambridge, Massachusetts: MIT Press.

Wolf, Reinhard \& Martin Heisenberg (1980): On the Fine Structure of Yaw Torque in Visual Flight Orientation of Drosophila melanogaster II. Visual Attention. In: Journal of Comparative Physiology A (140), S. 69-80.

Wolf, Reinhard \& Martin Heisenberg (1991): Basic Organization of Operant Behavior as Revealed in Drosophila Flight Orientation. In: Journal of Comparative Physiology A (169), S. 699-705. 